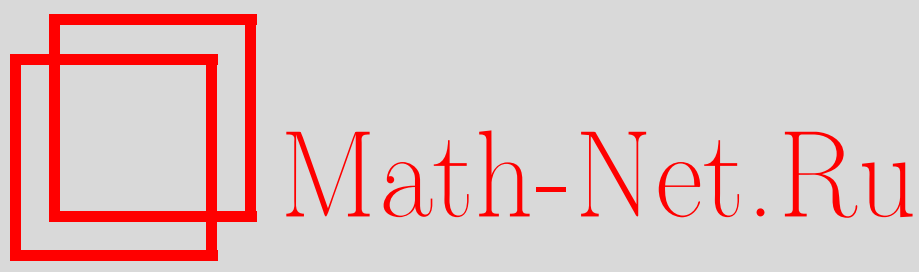

Л. С. Ефремова, Е. Н. Махрова, Динамика монотонных отображений дендритов, Матем. сб., 2001, том 192, номер 6, 15-30

DOI: https://doi.org/10.4213/sm570

Использование Общероссийского математического портала Math-Net.Ru подразумевает, что вы прочитали и согласны с пользовательским соглашением http://www . mathnet.ru/rus/agreement

Параметры загрузки:

IP : 54.147 .182 .235

26 апреля 2023 г., $14: 46: 47$ 
УДК 517.9

\author{
Л. С. Ефремова, Е.Н. Махрова
}

\title{
Динамика монотонных отображений дендритов
}

Рассмотрены монотонные отображения дендритов со счетньм замкнутьм множеством точек ветвления конечного порядка. Установлена структура $\omega$-предельных множеств, множеств периодических и неблуждающих точек; доказано, что топологическая энтропия монотонных отображений равна 0. Показано, что монотонные отображения дендритов с незамкнутым множеством точек ветвления конечного порядка могут иметь свойства, отличные от свойств монотонных отображений дендритов рассмотренного класса.

Библиограбоия: 15 названий.

\section{§1. Введение}

В настояшее время сушествует обширная библиография по непрерывным отображениям интервала, окружности, конечных графов. Естественное направление развития этих исследований связано с переходом к изучению динамических систем на разветвленных континуумах со счетным множеством точек ветвления. Однако здесь имеются лишш отдельные результаты. Так, в [1] доказана теорема существования неподвижной точки непрерывного отображения дендрита в себя; в [2] приведены некоторые оценки топологической энтропии; в [3] указан пример разветвленного континуума и такого немонотонного отображения на нем, центр которого не совпадает с замыканием множества периодических точек; в [4] исследована глубина центра; в [5] введено понятие простейшего отображения дендрита в себя и доказаны теоремы о структуре множеств периодических, $\omega$-предельных и неблуждающих точек.

Настоящая работа посвящена монотонным динамическим системам, заданным на дендритах и является продолжением статей [5] и [6].

Дендритом называется локально связный континуум (компактное связное метрическое пространство), не содержащий подмножеств, гомеоморфных окружности.

Будем рассматривать класс $D$ дендритов, обладаюших следующими свойствами: для любого $X \in D$

1) множество точек ветвления $R(X)$ замкнуто;

2) для произвольной точки $x \in R(X)$ число компонент множества $X \backslash\{x\}$ конечно.

Работа вьполнена при финансовой поддержке Российского фонда фундаментальных исследований (грант № 96-01-01755) и Министерства общего и профессионального образования России (грант № 97-0-1.8-109).

(С) Л.С. Етремова, Е.Н. Махрова 2001 
Сформулируем основные результаты. Так, следующая теорема А показывает, что неограниченность множества $T(f)$ периодов периодических точек монотонного отображения влечет за собой счетность множества точек ветвления дендрита.

Теорема А. Пусть $f$-монотонное отобрахсение дендрита $X \in D$ в себя, а множество $T(f)$ неограничено. Тогда множество $R(X)$ точек ветвления $X$ счетно.

В следующей теореме В описана структура множеств периодических, $\omega$-предельных и неблуждающих точек монотонных отображений дендритов, вычислена топологическая энтропия.

Tеорема В. Пусть $f$-монотонное отображсение дендрита $X \in D$. Тогда

(B1) множсество $\operatorname{Per}(f)$ периодических точек $f$ замкнуто;

(В2) для любой точки $x \in X \quad \omega$-предельное множество $\omega(x, f)$ траектории $\left\{f^{i}(x)\right\}_{i \geqslant 0}$ есть периодическая орбита;

(B3) $\operatorname{Per}(f)=\bigcup_{x \in X} \omega(x, f)=\Omega(f)=C(f)$, где $\Omega(f)$ - множсество неблужсдающих точек, $C(f)$ - чентр отображения $f$;

(B4) $h(f)=0$, где $h(f)-$ топологическая әнтропия $f$.

Следующая теорема С подчеркивает определяющую роль структуры множества точек ветвления дендрита в динамике монотонного отображения и устанавливает существование дендритов, не принадлежащих классу $D$, и таких монотонных отображений, которые не обладают свойствами (B1)-(В3).

Теорема C. Существуют дендрит $X \notin D$ и монотонное отображсние $f: X \rightarrow X$ maкue, чmo

(C1) множсество $\operatorname{Per}(f)$ периодических точек $f$ незамкнуто;

(C2) $f$ содержит рекуррентную непериодическую точку.

\section{§ 2. Предварительные сведения}

1. Сформулируем необходимые определения и напомним основные свойства дендритов (см. [7; 51, п. 6]).

Под дугой в дендрите $X$ будем понимать множество, гомеоморфное замкнутому промежутку на прямой (одноточечное множество будем считать вырожденной дугой).

Свойство 2.1 (дугообразной связности). Любие две точки $x, y, x \neq y$, в дендрите $X$ можсно соединить единственной дугой, имеющей в качестве концов точки $х$ и $y$.

В дальнейшем символом $\gamma(x, y)$ будем обозначать дугу с концами в точках $x$ и $y$, содержащую эти точки.

ОПРЕДЕЛЕНИЕ 2.2. Пусть $X$ - произвольньй континуум, а $n$ - порядковое число $\omega$ множества целых неотрицательных чисел в их естественном порядке или кардинальное число $\leqslant c$ ( $c$ - мощность континуума $)$. Говорят, что порядок точки $x \in X$ не превосходит $n(\operatorname{ord} x \leqslant n)$, если для любого $\varepsilon>0$ сушествует $0<\delta<\varepsilon$ такое, что $\operatorname{card}\left(\partial U_{\delta}(x)\right) \leqslant n$, где $\partial(\cdot)-$ граница множества, $\operatorname{card}(\cdot)-$ мощность 
множества. Равенство ord $x=n$ означает, что ord $x \leqslant n$ и соотношение $\operatorname{ord} x \leqslant m$ не имеет места ни при каком $m \geqslant n$.

Свойство 2.3 (регулярности). Всякий дендрит X есть регулярный континуум, т.е. любая точка $x \in X$ имеет порядок $\leqslant \omega$.

Из свойства 2.3 следует, что каждњй подконтинуум дендрита есть дендрит.

Теорема 2.4. Пусть дендрит $X$ содержит более одной точки. Тогда в $X$ существуют точки первого порядка, называемье концевыми; всюду плотнь точки второго порядка; существует не более чем счетное множество точек ветвления (точек порядка, большего 2).

ТеОРема 2.5. Пусть $X$ - дендрит. Если число компонент множества $X \backslash\{x\}$ конечно, то оно совпадает $c$ ord $x$.

Из теорем 2.4 и 2.5 следует, что если множество точек ветвления $R(X)$ дендрита $X \in D$ непусто, то $R(X)$ - разреженное множество, и для любого $z \in R(X)$ число компонент множества $X \backslash\{z\}$ совпадает с ord $z$.

Условимся обозначать через $X_{i}(z), 1 \leqslant i \leqslant \operatorname{ord} z$, компоненты множества $X \backslash\{z\}$.

Свойство 2.6. Пусть $X$ - дендрит, а $Y$ - собственный подконтинуум $X$. Тогда любая компонента $U$ открытого множества $X \backslash Y$ имеет в $X$ единственную граничную точку $u \in Y \quad(\partial U=u)$.

2. В этой части работы мы докажем основные свойства монотонных отображений.

ОПРЕДЕЛЕНИЕ 2.7 [7; $§ 46$, п. 1]. Отображение $f: X \rightarrow X$ называется монотонныц, если $f$ непрерывно и полный прообраз произвольного связного подмножества из $f(X)$ связен.

ОПРЕДЕЛЕНИЕ 2.8 [5]. Отображение $f: X \rightarrow X$ назьвается простейшим, если $f$ непрерывно и полный прообраз любой дуги из $f(X)$ есть дуга.

Обозначим $M^{0}(X)\left(P^{0}(X)\right)$ множество всех монотонных (простейших) отображений дендрита $X \in D$ в себя. Заметим, что $P^{0}(X) \subset M^{0}(X)$, но $P^{0}(X) \neq$ $M^{0}(X)$. Для любого $f \in M^{0}(X)$ и для каждого $i \geqslant 1 f^{i} \in M^{0}(X)$.

Условимся обозначать через $f^{-i}(A), i \geqslant 1, i$-й полньй прообраз произвольного множества $A \subset f(X)$; положим $f^{-0}(A)=A$.

Перейдем к доказательству основных свойств монотонных отображений дендритов.

Лемма 2.9. Если $f \in M^{0}(X)$, то для произвольных точек $x, y \in X$ справедливо равенство $f(\gamma(x, y))=\gamma(f(x), f(y))$.

ДокАЗАтЕльство. Предположим, что $f(\gamma(x, y))$ не является дугой. Тогда $f(\gamma(x, y))$ - дендрит с непустым множеством точек ветвления и найдется точка $z \in \gamma(x, y) \backslash\{x, y\}$ такая, что справедливо

$$
f(z) \text { - концевая точка } f(\gamma(x, y)) \text {. }
$$


При этом выполнено свойство $(f(\gamma(x, z)) \cap f(\gamma(y, z))) \backslash\{f(z)\} \neq \varnothing$. Отсюда получаем, что полный прообраз любого связного множества $A \subset(f(\gamma(x, z)) \cap$ $f(\gamma(y, z))) \backslash\{f(z)\}$ не связен. Таким образом, сделанное предположение неверно и $f(\gamma(x, y))$ - дуга. Из предыдушего следует также, что если для некоторой точки $z \in \gamma(x, y) \backslash\{x, y\}$ выполнено свойство $(*)$, то либо $f(z)=f(x)$, либо $f(z)=f(y)$. Лемма 2.9 доказана.

Лемма 2.9 показывает, что действие монотонного отображения на дендрите согласовано со свойством дугообразной связности. Для сравнения отметим, что монотонные отображения на наследственно неразложимых континуумах могут одномерные подмножества континуума отображать на бесконечномерные [8].

Лемма 2.10. Пусть $f \in M^{0}(X)$. Тогда для любой точки $z \in X$ такой, что ord $z \geqslant 2$, и различныи $1 \leqslant i_{1}, i_{2} \leqslant \operatorname{ord} z \quad f\left(\overline{X_{i_{1}}(z)}\right) \cap f\left(\overline{X_{i_{2}}(z)}\right)=\{f(z)\}$.

ДокАЗАТЕЛЬСтво. Предположим противное. Тогда найдутся точка $z \in X$ $(\operatorname{ord} z \geqslant 2)$ и две ее различные компоненты $X_{i_{1}}(z), X_{i_{2}}(z)$ такие, что $f\left(\overline{X_{i_{1}}(z)}\right) \cap$ $f\left(\overline{X_{i_{2}}(z)}\right) \neq\{f(z)\}$. Так как последнее пересечение не пусто, то сушествует точка $x \in f\left(\overline{X_{i_{1}}(z)}\right) \cap f\left(\overline{X_{i_{2}}(z)}\right), x \neq f(z)$. Тогда $f^{-1}(x)$ - несвязное множество. Последнее противоречит монотонности $f$. Лемма 2.10 доказана.

СлЕДСтвиЕ 2.11. Пусть $f \in M^{0}(X) . \quad$ Если $z \in R(X), f(z) \notin R(X), m o$ существует не менее $(\operatorname{ord} z-2)$ компонент точки $z$, которые отображаются в $f(z)$.

Действительно, так как $\operatorname{ord} z \geqslant 3$, a ord $f(z) \leqslant 2$, то в силу леммы 2.10 не менее (ord $z-2$ ) компонент точки $z$ должны отобразиться в $f(z)$.

Лемма 2.12. Пусть $f \in M^{0}(X), z^{0} \in \operatorname{Per}(f), m \geqslant 1-$ период $z^{0}$. Тогда следующие утверждения әквивалентны:

1) существует дуга в $X$, содержащая $\operatorname{Orb}\left(z^{0}, f\right)$, где

$$
\operatorname{Orb}\left(z^{0}, f\right)=\left\{z^{0}, \ldots, z^{m-1}\right\}
$$

$$
z^{i}=f^{i}\left(z^{0}\right), 0 \leqslant i \leqslant m-1
$$

2) $m=1$ или 2 .

Доказательство леммы 2.12 имеется в [5].

Пусть $\gamma(x, y)$-произвольная невырожденная дуга в $X, z_{1}, z_{2}$ - различные точки из $\gamma(x, y)$, а $\varphi$ - гомеоморфизм отрезка $[0,1]$ на $\gamma(x, y)$. Следуя [5], будем говорить, что $z_{1} \prec_{\varphi} z_{2}\left(z_{1} \varphi\right.$-nредшествует $\left.z_{2}\right)$, если $\varphi^{-1}\left(z_{1}\right)<\varphi^{-1}\left(z_{2}\right)$, где "<" - естественный порядок на отрезке $[0,1]$.

Лемма 2.13. Пусть $f \in M^{0}(X), z^{0} \in \operatorname{Per}(f), m \geqslant 1-$ период точки $z^{0}$. Если $x^{0} \in \operatorname{Per}(f), x^{0} \notin \operatorname{Orb}\left(z^{0}, f^{m}\right)$, то любая невырожденная дуга $\gamma\left(z^{0}, z\right)$ содержит не более одной точки $\operatorname{Orb}\left(x^{0}, f^{m}\right)$.

ДокАЗАТЕльство. Предположим противное. Тогда сушествует невырожденная дуга $\gamma\left(z^{0}, z\right)$, содержащая не менее двух различных точек $\operatorname{Orb}\left(x^{0}, f^{m}\right)$. Пусть точка $y^{0} \in \operatorname{Orb}\left(x^{0}, f^{m}\right) \cap \gamma\left(z^{0}, z\right)$ такова, что при некотором $m_{0} \geqslant 1 y^{m m_{0}}=$ 
$f^{m m_{0}}\left(y^{0}\right) \in \gamma\left(z^{0}, y^{0}\right) \backslash\left\{z^{0}, y^{0}\right\}$, а гомеоморфизм $\varphi:[0,1] \rightarrow \gamma\left(z^{0}, y^{0}\right)$ удовлетворяет следуюшим условиям: $\varphi(0)=z^{0}, \varphi(1)=y^{0}$. Тогда в силу леммы 2.9 дуга $\gamma\left(z^{0}, y^{0}\right)$ инвариантна относительно $f^{m m_{0}}$ и $z^{0} \prec_{\varphi} \cdots \prec_{\varphi} y^{(j+1) m m_{0}} \prec_{\varphi}$ $y^{j m m_{0}} \prec_{\varphi} \cdots \prec_{\varphi} y^{m m_{0}} \prec_{\varphi} y^{0}, j=0,1,2, \ldots$ Последнее противоречит тому, что $y^{0} \in \operatorname{Per}(f)$. Лемма 2.13 доказана.

Лемма 2.14. Пусть $f \in M^{0}(X), z^{0} \in \operatorname{Per}(f) \cap R(X), m \geqslant 1-$ nериод $z^{0}$. Тогда $\bigcup_{i=0}^{+\infty} f^{-m i}\left(z^{0}\right)-$ связное множсество.

ДокАЗАтЕльство. Утверждение справедливо, если $\bigcup_{i=0}^{+\infty} f^{-m i}\left(z^{0}\right)$ - одноточечное множество. Предположим, что $\bigcup_{i=0}^{+\infty} f^{-m i}\left(z^{0}\right)$ содержит более одной точки. Возьмем произвольно две различные точки $x, y \in \bigcup_{i=0}^{+\infty} f^{-m i}\left(z^{0}\right)$. Тогда существуют натуральные числа $i(x)$ и $i(y)$ такие, что $f^{i(x) m}(x)=f^{i(y) m}(y)=z^{0}$. Обозначим $i_{0}=\max \{i(x), i(y)\}$. Так как $f^{m}\left(z^{0}\right)=z^{0}$, то $x, y \in f^{-i_{0} m}\left(z^{0}\right)$. В силу монотонности $f$ множество $f^{-i_{0} m}\left(z^{0}\right)$ связно. Следовательно, справедливо включение $\gamma(x, y) \subset f^{-i_{0} m}\left(z^{0}\right)$. Поэтому $\gamma(x, y) \subset \bigcup_{i=0}^{+\infty} f^{-m i}\left(z^{0}\right)$, т.е. множество $\bigcup_{i=0}^{+\infty} f^{-m i}\left(z^{0}\right)$ дугообразно связно. Лемма 2.14 доказана.

СлеДСтвиЕ 2.15. Пусть $f \in M^{0}(X), z^{0} \in \operatorname{Per}(f) \cap R(X), m \geqslant 1$ - период точки $z^{0}$. Тогда $\overline{\bigcup_{i=0}^{+\infty} f-m i\left(z^{0}\right)}$ - дендрит; если множество $\bigcup_{i=0}^{+\infty} f^{-m i}\left(z^{0}\right)$ не замкнуто, то каждая точка из $\overline{\bigcup_{i=0}^{+\infty} f^{-m i}\left(z^{0}\right)} \backslash \bigcup_{i=0}^{+\infty} f^{-m i}\left(z^{0}\right)$ является концевой точкой континуума $\overline{\bigcup_{i=0}^{+\infty} f^{-m i}\left(z^{0}\right)}$ и множество $\overline{\bigcup_{i=0}^{+\infty} f^{-m i}\left(z^{0}\right)}$ $\bigcup_{i=0}^{+\infty} f^{-m i}\left(z^{0}\right)$ инвариантно относительно $f^{m}$.

Лемма 2.16. Пусть $f \in M^{0}(X), z \in R(X) \backslash \operatorname{Per}(f)$. Тогда не более двух компонент точки z содержст периодические точки $f$.

Доказательство леммы 2.16 приведено в [5].

Завершая эту часть работы, отметим, что монотонные динамические системы находят широкое применение в обшей теории динамических систем (см., например, [9]).

\section{§3. Доказательство теоремы А}

Пусть $X$ - конечное дерево, а монотонное отображение $f: X \rightarrow X$ таково, что множество точек ветвления $R(X)$ инвариантно относительно $f$. Тогда для любой точки $z \in R(X) \backslash \operatorname{Per}(f)$ существует точка $z^{0} \in \operatorname{Per}(f) \cap R(X)$ такая, что $z \in$ $\bigcup_{i=0}^{+\infty} f^{-m i}\left(z^{0}\right)$, где $m \geqslant 1-$ период $z^{0}$.

Положим $K(z)=\left\{X_{j}(z), 1 \leqslant j \leqslant \operatorname{ord} z: X_{j}(z) \subset \bigcup_{i=0}^{+\infty} f^{-m i}\left(z^{0}\right)\right\}$.

Для обоснования корректности определения множества $K(z)$ опишем динамику монотонного отображения на множестве концевых точек континуума $\overline{\bigcup_{i=0}^{+\infty} f^{-m i}\left(z^{0}\right)}$

Лемма 3.1. Пусть $f \in M^{0}(X), X$ - конечное дерево, существует точка $z^{0} \in \operatorname{Per}(f) \cap R(X)$ такая, что $\bigcup_{i=0}^{+\infty} f^{-m i}\left(z^{0}\right) \neq\left\{z^{0}\right\}$, где $m \geqslant 1-$ период $z^{0}$. Тогда

1) какова бын ни была кониевая точка е континуума $\overline{\bigcup_{i=0}^{+\infty} f-m i\left(z^{0}\right)}$, справедливо либо е $\in \bigcup_{i=0}^{+\infty} f^{-m i}\left(z^{0}\right)$, либо $е \in \operatorname{Per}(f) \backslash\left\{z^{0}\right\}$;

2) если существует концевая точка е, не являющаяся концевой точкой $X$, mо $e \in \operatorname{Per}(f)$. 
ДокаЗАТЕЛЬСтво. 1. Убедимся в справедливости утверждения 1). Какова бы ни была концевая точка $e \in \overline{\bigcup_{i=0}^{+\infty} f^{-m i}\left(z^{0}\right)}$, возможно либо $e \in \bigcup_{i=0}^{+\infty} f^{-m i}\left(z^{0}\right)$, либо $e \in \overline{\bigcup_{i=0}^{+\infty} f^{-m i}\left(z^{0}\right)} \backslash \bigcup_{i=0}^{+\infty} f^{-m i}\left(z^{0}\right)$. Рассмотрим вторую из указанных возможностей. Тогда $e \neq z^{0}$. В силу следствия 2.15 множество $\overline{\bigcup_{i=0}^{+\infty} f-m i\left(z^{0}\right)}$ - конечное дерево. Поэтому траектория $\left\{f^{m i}(e)\right\}_{i \geqslant 0}$ точки $e$ состоит из конечного числа попарно различных точек. Отсюда получаем, что либо $e \in \operatorname{Per}(f)$, либо $e \notin \operatorname{Per}(f)$, но при некотором $j \geqslant 1 e_{j}=f^{m j}(e)$ - периодическая точка относительно $f^{m}$. Исключим последнюю возможность. Действительно, в противном случае найдутся различные точки $z_{1}=f^{m(j-1)}(e)$ и $z_{2}=f^{m(j+k-1)}(e)$ $\left(k \geqslant 1\right.$ - период $e_{j}$ относительно $\left.f^{m}\right)$ такие, что $f^{m}\left(z_{1}\right)=f^{m}\left(z_{2}\right)=e_{j}$. Так как $f^{m}$ - монотонное отображение, то $\gamma\left(z_{1}, z_{2}\right) \subset f^{-m}\left(e_{j}\right)$. С другой стороны, в силу следствия $2.15 \gamma\left(z_{1}, z_{2}\right)$ содержит точки $\bigcup_{i=0}^{+\infty} f^{-m i}\left(z^{0}\right)$. Следовательно, $e \in \bigcup_{i=0}^{+\infty} f^{-m i}\left(z^{0}\right)$. Последнее невозможно. Следовательно, $e \in \operatorname{Per}(f)$.

2. Докажем утверждение 2). Если $e=z^{0}$, то утверждение 2) справедливо. Пусть $e \neq z^{0}$. Тогда $\overline{\bigcup_{i=0}^{+\infty} f^{-m i}\left(z^{0}\right)} \neq X$ и найдется компонента $U$ открытого множества $X \backslash \overline{\bigcup_{i=0}^{+\infty} f-m i\left(z^{0}\right)}$, которая имеет в $X$ единственную граничную точку $е$. Предположим, что $e \in \bigcup_{i=0}^{+\infty} f^{-m i}\left(z^{0}\right)$. Тогда сушествует $m_{0} \geqslant 1$ такое, что $f^{m m_{0}}(e)=z^{0}$ и при любом $n \geqslant m_{0} \overline{f^{n m}(U)}-$ конечное дерево, содержащее более одной точки и имеющее конщевую точку $z^{0}$. Следовательно, для любого $n \geqslant m_{0}$ справедливо равенство $\overline{\left(f^{n m}(U)\right)} \cap\left(\bigcup_{i=0}^{+\infty} f^{-m i}\left(z^{0}\right)\right)=\left\{z^{0}\right\}$. Таким образом, в силу следствия 2.15 сушествует $f^{m}$-инвариантное дерево $X^{*}=$ $\overline{\bigcup_{l=1}^{p} X_{j_{l}}}\left(z^{0}\right)$, где $X_{j_{1}}\left(z^{0}\right), X_{j_{2}}\left(z^{0}\right), \ldots, X_{j_{p}}\left(z^{0}\right)$ - компоненты точки $z^{0}$ такие, что $X_{j_{l}}\left(z^{0}\right) \cap \overline{\bigcup_{i=0}^{+\infty} f^{-m i}\left(z^{0}\right)}=\varnothing, X_{j_{l}}\left(z^{0}\right) \cap \overline{\bigcup_{n=m_{0}}^{+\infty} f^{n m}(U)} \neq \varnothing$ для любого $1 \leqslant l \leqslant p$. Более того, $\bar{U} \cap X^{*}=\varnothing$. Отсюда следует существование двух различных компонент точки $z^{0} \quad X_{j_{l_{0}}}\left(z^{0}\right)\left(1 \leqslant l_{0} \leqslant p\right)$ и $X_{j_{s}}\left(z^{0}\right)\left(p<s \leqslant \operatorname{ord} z^{0}\right)$ таких, что $U \subset X_{j_{s}}\left(z^{0}\right)$ и для некоторого $n_{0} \geqslant m_{0} f^{m n_{0}}\left(\overline{X_{j_{l_{0}}}\left(z^{0}\right)}\right) \cap f^{m n_{0}}\left(\overline{X_{j_{s}}\left(z^{0}\right)}\right) \neq\left\{z^{0}\right\}$. Полученное противоречие с леммой 2.10 доказьвает, что $e \notin \bigcup_{i=0}^{+\infty} f^{-m i}\left(z^{0}\right)$. В силу утверждения 1) $e \in \operatorname{Per}(f)$. Следовательно, утверждение 2) доказано. Доказательство леммы 3.1 завершено.

Перейдем к обоснованию корректности определения множества $K(z)$.

Лемма 3.2. Пусть $f \in M^{0}(X)$. Существуют точки $z^{0} \in \operatorname{Per}(f) \cap R(X)$, $z \in R(X) \backslash \operatorname{Per}(f)$ mакие, что $f^{m}(z)=z^{0}$, где $m \geqslant 1-$ период $z^{0}$. Тогда существует не менее (ord $z-2)$ компонент точки $z$, принадлехсащих множсеству $\bigcup_{i=0}^{+\infty} f^{-m i}\left(z^{0}\right)$.

ДокАЗАтЕльство. В силу леммы 2.16 сушествует не менее (ord $z-2$ ) компонент $\left\{X_{j_{s}}(z)\right\}, 1 \leqslant s \leqslant$ ord $z-2$, которые не содержат периодических точек $f$. Покажем, что $X_{j_{s}}(z) \subset \bigcup_{i=0}^{+\infty} f^{-m i}\left(z^{0}\right)$ для любого $1 \leqslant s \leqslant \operatorname{ord} z-2$. Действительно, в противном случае найдется конщевая точка $е$ континуума $\overline{\bigcup_{i=0}^{+\infty} f-m i\left(z^{0}\right)}$ такая, что $e \in X_{j_{s_{0}}}(z)$ для некоторого $1 \leqslant s_{0} \leqslant$ ord $z-2$ и $е$ не является концевой точкой $X$. Тогда в силу утверждения 2) леммы $3.1 e \in \operatorname{Per}(f)$, что противоречит выбору компоненты $X_{j_{s_{0}}}(z)$. Лемма 3.2 доказана.

Таким образом, множество $K(z)$ состоит из целых компонент. 
Следующая теорема 3.3 устанавливает структуру монотонных отображений конечных деревьев и является следствием лемм 3.1 и 3.2 .

Teоpema 3.3. Пусть $f \in M^{0}(X), X$ - конечное дерево. Тогда

1) если $f(R(X)) \subset R(X) u\{z \in X \backslash R(X): f(z) \in f(R(X))\}=\varnothing, m o$ $f(R(X))=R(X)$ u $f \in P^{0}(X)$;

2) если $f(R(X)) \subset R(X),\{z \in X \backslash R(X): f(z) \in f(R(X))\} \neq \varnothing$, то мнохество $Y=\overline{X \backslash \bigcup_{z^{0} \in \operatorname{Per}(f) \cap R(X)} \bigcup_{i=0}^{+\infty} f^{-m i}\left(z^{0}\right)}$ инвариантно; более того, если $Y \neq \varnothing$, то существуют $f$-инвариантное дерево $X^{*}=X \backslash \bigcup_{z \in R(X) \backslash \operatorname{Per}(f)} K(z)$ и отображсение $g \in P^{0}\left(X^{*}\right)$ такие, что $R\left(X^{*}\right) \backslash \operatorname{Per}(f)=\left.\varnothing u f\right|_{Y}=\left.g\right|_{Y}$, әде $\left.(\cdot)\right|_{Y}-$ сужение отображсения $(\cdot)$ на $Y$;

3) если $f(R(X)) \not \subset R(X)$, то существует натуральное число $k \geqslant 1$ такое, что $f^{k}(X)$ либо $f$-инвариантная дуга, либо дерево с непустым множсеством точек ветвления; в последнем случае множество точек ветвления дерева $f^{k}(X)$ инвариантно относительно $f$.

Заметим, что если $X-n$-од, т.е. $X=\left\{z \in \mathbb{C}: z^{n} \in[0,1]\right\}$, где $\mathbb{C}$ - комплексная плоскость, $n \geqslant 3$, и $f(R(X)) \not \subset R(X)$, то $k=1$, а $f(X)$ - $f$-инвариантная дуга [6].

Теорема 3.3 показывает, что арифметические соотношения между периодами периодических точек монотонных отображений конечных деревьев совпадают с арифметическими соотношениями между периодами периодических точек простейших отображений, описание которых дано в [5].

ОПРЕДЕЛЕНИЕ 3.4. Конечное упорядоченное множество натуральных чисел $M=\left\{m_{1}, m_{2}, \ldots, m_{r}\right\}$, где $m_{1}<m_{2}<\cdots<m_{r}$ при $r>1$, будем называть допустимым, если сушествует монотонное отображение $f n$-ода $X(n \geqslant 3)$ в себя такое, что $f(0)=0$, а множество периодов периодических точек $f$ совпадает с $M$.

Таким образом, множество $M=\left\{m_{1}, m_{2}, \ldots, m_{r}\right\}$, где $m_{1}<m_{2}<\cdots<m_{r}$ для $r>1$, допустимо в смысле определения 3.4 тогда и только тогда, когда $m_{1}=1$ и $\sum_{j=2}^{r} m_{j} \leqslant n$. Укажем также, что $\operatorname{card} M \leqslant E\left(\frac{-1+\sqrt{9+8 n}}{2}\right)$, где $E(\cdot)-$ целая часть числа $(\cdot)$ [5].

Положим $Z=X$ в случае 1$)$ теоремы $3.3 ; Z=X^{*}$ в случае 2$)$ теоремы 3.3 ; $Z=f^{k}(X)$ в случае 3$)$ теоремы 3.3 , когда $\left.f\right|_{f^{k}(X)} \in P^{0}(X) ; Z=f^{k}(X) \backslash$ $\bigcup_{z \in R\left(f^{k}(X)\right) \backslash \operatorname{Per}(f)} K(z)$ в случае 3$)$ теоремы 3.3 , когда $\left\{z \in f^{k}(X) \backslash R\left(f^{k}(X)\right)\right.$ : $\left.f(z) \in f\left(R\left(f^{k}(X)\right)\right)\right\} \neq \varnothing$.

Пусть множество периодов периодических точек сужения отображения $f$ на $R(Z) T\left(\left.f\right|_{R(Z)}\right)$ содержит натуральные числа $m_{1}, m_{2}, \ldots, m_{\nu}, \nu \geqslant 1$, и для $\nu>1$ $m_{1}<m_{2}<\cdots<m_{\nu}$. Обозначим через $z^{0}\left(m_{j}\right)$ точку множества $R(Z)$ с периодом $m_{j}, 1 \leqslant j \leqslant \nu$. Пусть $Z^{(n)}\left(z^{0}\left(m_{j}\right)\right)$ - континуум со следующими свойствами.

1. $Z^{(n)}\left(z^{0}\left(m_{j}\right)\right)$ имеет единственную точку ветвления $z^{0}\left(m_{j}\right)$ и гомеоморфен $n$-оду при $n=$ ord $z^{0}\left(m_{j}\right)$;

2. Если $R(Z) \cap Z_{i}\left(z^{0}\left(m_{j}\right)\right)=\varnothing$, то компонента $Z_{i}\left(z^{0}\left(m_{j}\right)\right), 1 \leqslant i \leqslant \operatorname{ord} z^{0}\left(m_{j}\right)$, множества $Z \backslash\left\{z^{0}\left(m_{j}\right)\right\}$ является также компонентой множества $Z^{(n)}\left(z^{0}\left(m_{j}\right)\right) \backslash$ $\left\{z^{0}\left(m_{j}\right)\right\}$. В противном случае компонентой $Z^{(n)}\left(z^{0}\left(m_{j}\right)\right) \backslash\left\{z^{0}\left(m_{j}\right)\right\}$ служит дуга 
$Z_{i}^{(n)}\left(z^{0}\left(m_{j}\right)\right)$ из $Z_{i}\left(z^{0}\left(m_{j}\right)\right)$ такая, что $\operatorname{card}\left(R(Z) \cap \overline{Z_{i}^{(n)}\left(z^{0}\left(m_{j}\right)\right)}\right)=2$; концевыми точками $Z_{i}^{(n)}\left(z^{0}\left(m_{j}\right)\right)$ являются точки из $R(Z)$, причем одна из них $-z^{0}\left(m_{j}\right)$.

В силу лемм $2.10,3.1$ и 3.2 справедливо $\left.f^{m_{j}}\right|_{Z^{(n)}\left(z^{0}\left(m_{j}\right)\right)} \in M^{0}\left(Z^{(n)}\left(z^{0}\left(m_{j}\right)\right)\right)$. Следовательно, допустимое множество $M\left(z^{0}\left(m_{j}\right)\right)$, соответствующее континууму $Z^{(n)}\left(z^{0}\left(m_{j}\right)\right)\left(n=\operatorname{ord} z^{0}\left(m_{j}\right)\right)$, определено корректно и может быть использовано для описания арифметических соотношений между периодами периодических точек монотонных отображений конечных деревьев.

Tеорема 3.5. Пусть $f \in M^{0}(X), X$-конечное дерево. Если $R(Z) \neq \varnothing$, то 1) $m_{1}<3$;

2) при $\nu>1$ существует допустимое мнохсество $M\left(z^{0}\left(m_{j}\right)\right), 1 \leqslant j \leqslant$ $\nu-1$, соответствующее некоторому континууму $Z^{(n)}\left(z^{0}\left(m_{j}\right)\right)$, такое, что

$$
m_{j+1} \in m_{j} \cdot M\left(z^{0}\left(m_{j}\right)\right)
$$

3) для любой компоненты $Z_{i}\left(z^{0}\left(m_{j}\right)\right), 1 \leqslant i \leqslant \operatorname{ord} z^{0}\left(m_{j}\right)$, множества $Z \backslash\left\{z^{0}\left(m_{j}\right)\right\}$ без точек ветвления и произвольной точки $z \in \operatorname{Per}(f) \cap$ $Z_{i}\left(z^{0}\left(m_{j}\right)\right)$ периода $m=m(z), 1 \leqslant j \leqslant \nu$, существует допустимое мнохсество $M\left(z^{0}\left(m_{j}\right)\right)$, соответствующее некоторому континууму $Z^{(n)}\left(z^{0}\left(m_{j}\right)\right)$, такое, что

$$
m \in m_{j} \cdot M\left(z^{0}\left(m_{j}\right)\right)
$$

Укажем, что если $R(Z)=\varnothing$, то $f$ содержит только неподвижные точки и, быть может, периодические точки периода два.

Справедливость теоремы 3.5 следует из теоремы 3.3.

СлеДСТВИЕ 3.6. Если $f \in M^{0}(X), X$ - конечное дерево, то множество периодов периодических точек отображсени $f$ ограничено.

Таким образом, если $f \in M^{0}(X)$ и $T(f)$ - неограниченное множество, то множество $R(X)$ счетно. Теорема А доказана.

\section{§4. Доказательство теоремы В}

1. В этой части работы мы докажем утверждение (B1) теоремы В.

Обозначим через $\operatorname{Fix}(f)$ множество неподвижных точек отображения $f$, через $\operatorname{Pois}(f)$ - множество точек, устойчивых по Пуассону.

Teopema 4.1. Пyсmь $f \in M^{0}(X)$. Tогдa $\operatorname{Pois}(f)=\operatorname{Per}(f)$.

ДокаЗАтельство. Предположим противное. Тогда сушествует точка $z \in$ $\operatorname{Pois}(f) \backslash \operatorname{Per}(f)$. Покажем, что найдется натуральное $n_{0} \geqslant 1$ такое, что $f^{n_{0}}(z) \notin$ $R(X)$. Действительно, в противном случае для траектории точки $z$ справедливо включение $\left\{f^{n}(z)\right\}_{n \geqslant 1} \subset R(X)$. Так как множество $R(X)$ замкнуто, то получаем отсюда, что ${\overline{\left\{f^{n}(z)\right\}_{n}}}_{n 1} \subset R(X)$, т.е. множество $R(X)$ имеет мощность континуума. Последнее противоречит теореме 2.4. Таким образом, при некотором натуральном $n_{0} \geqslant 1 f^{n_{0}}(z) \notin R(X)$. Замкнутость множества $R(X)$ влечет за собой существование окрестности $U\left(f^{n_{0}}(z)\right)$ точки $f^{n_{0}}(z)$ такой, что 
$U\left(f^{n_{0}}(z)\right) \cap R(X)=\varnothing$. Так как $f^{n_{0}}(z) \in \operatorname{Pois}(f) \backslash \operatorname{Per}(f)$, то существует натуральное $n_{1} \geqslant 1$, при котором $f^{n_{0}+n_{1}}(z) \in U\left(f^{n_{0}}(z)\right) \backslash\left\{f^{n_{0}}(z)\right\}$. Положим $y=f^{n_{0}}(z), g=f^{n_{1}}$. В силу леммы $2.9 g(\gamma(y, g(y)))=\gamma\left(g(y), g^{2}(y)\right)$. Так как $\gamma(y, g(y)) \cap R(X)=\varnothing$, то реализуется один из следующих случаев:

1) $g(y) \in \gamma\left(y, g^{2}(y)\right) \backslash\left\{y, g^{2}(y)\right\}$;

2) $g^{2}(y) \in \gamma(y, g(y)) \backslash\{y, g(y)\}$;

3) $y \in \gamma\left(g(y), g^{2}(y)\right) \backslash\left\{g(y), g^{2}(y)\right\}$.

Рассмотрим случай 1). Тогда при любом $j \geqslant 1 g^{j+1}(y) \in \gamma\left(g^{j}(y), g^{j+2}(y)\right)$. Отсюда следует, что найдется дуга $\gamma(y, x)$ такая, что $\left\{g^{j}(y)\right\}_{j \geqslant 1} \subset \gamma(y, x)$. Зададим гомеоморфизм $\varphi:[0,1] \rightarrow \gamma(y, x)$ так, чтобы $\varphi(0)=y, \varphi(1)=x$. Тогда $y \prec_{\varphi} g(y) \prec_{\varphi} \ldots \prec_{\varphi} g^{j}(y) \prec_{\varphi} \ldots$. Последнее означает, что $y \notin \operatorname{Pois}(g)$. Следовательно, $f^{n_{0}}(z) \notin \operatorname{Pois}(f)$, и случай 1$)$ не реализуется.

Пусть реализуется случай 2). Тогда $g(\gamma(y, g(y)))$ - собственное подмножество $\gamma(y, g(y))$. Следовательно, $\left\{g^{j}(y)\right\}_{j \geqslant 1} \subset \gamma\left(g(y), g^{2}(y)\right)$. Так как $y \notin$ $\gamma\left(g(y), g^{2}(y)\right)$, то из последнего включения следует, что $y \notin \operatorname{Pois}(g)$. Таким образом, случай 2) невозможен.

Рассмотрим случай 3). Имеем: $\gamma(y, g(y)) \subset g(\gamma(y, g(y)))=\gamma\left(g(y), g^{2}(y)\right)$. Так как $\gamma(y, g(y)) \cap R(X)=\varnothing$ и $g \in M^{0}(X)$, то отсюда следует, что $\operatorname{Fix}\left(\left.g\right|_{\gamma(y, g(y))}\right) \neq \varnothing$. Пусть $\bar{z} \in \operatorname{Fix}(g) \cap \gamma(y, g(y))$, причем $\gamma(y, \bar{z}) \cap \operatorname{Fix}(g)=\{\bar{z}\}$. Положим $\tau=g^{2}$. Тогда $y \in \gamma(\bar{z}, \tau(y))$. Поэтому $\tau(y) \in \tau(\gamma(\bar{z}, \tau(y)))=\gamma\left(\bar{z}, \tau^{2}(y)\right)$. Отсюда следует, что $\tau(y) \in \gamma\left(y, \tau^{2}(y)\right)$ и $y \notin \operatorname{Pois}(\tau)$. Таким образом, случай 3$)$ также не реализуется. Следовательно, сделанное предположение не верно и $\operatorname{Pois}(f)=\operatorname{Per}(f)$. Теорема 4.1 доказана.

Нам потребуется также следующее вспомогательное утверждение.

Лемма 4.2. Пусть $f \in M^{0}(X)$, последовательность периодических точек $\left\{z_{j}\right\}_{j \geqslant 1}$ такова, что $\lim _{j \rightarrow \infty} z_{j}=z^{0}$ и все точки $z_{j}, j \geqslant 1$, лежст на некоторой компоненте $X_{k_{1}}\left(z^{0}\right)$ точки $z^{0}$. Тогда существует последовательность $\left\{x_{i}\right\}_{i \geqslant 1} \subset \operatorname{Per}(f) \cap X_{k_{1}}\left(z^{0}\right)$, все точки которой принадлежат дуге $\gamma\left(z^{0}, z_{1}\right)$, причем $\lim _{j \rightarrow \infty} x_{i}=z^{0}$.

ДокАЗАТЕЛЬство. Утверждение справедливо, если существует подпоследовательность $\left\{z_{j_{i}}\right\}_{i \geqslant 1} \subset\left\{z_{j}\right\}_{j \geqslant 1}$ такая, что при любом $j \geqslant 1 z_{j_{i}} \in \gamma\left(z^{0}, z_{1}\right)$. Тогда полагаем $x_{i}=z_{j_{i}}, i \geqslant 1$.

Рассмотрим случай, когда такой подпоследовательности не существует. Так как $\lim _{j \rightarrow \infty} z_{j}=z^{0}$, то найдется натуральное $j_{0} \geqslant 2$ такое, что при любом $j \geqslant j_{0}$ множество $\gamma\left(z^{0}, z_{j}\right) \cup \gamma\left(z_{j}, z_{1}\right)$ гомеоморфно 3 -оду. Обозначим $x_{j}$ точку ветвления $\gamma\left(z^{0}, z_{j}\right) \cup \gamma\left(z_{j}, z_{1}\right)$. Тогда три различные компоненты точки $x_{j}$ содержат периодические точки $f$. В силу леммы $2.16 x_{j} \in \operatorname{Per}(f)$. Таким образом, при любом $j \geqslant j_{0}$ существует точка $x_{j} \in \operatorname{Per}(f) \cap \gamma\left(z^{0}, z_{1}\right)$. Из сходимости последовательности $\left\{z_{j}\right\}_{j \geqslant 1} \mathrm{k} z^{0}$ следует, что $\lim _{j \rightarrow \infty} x_{j}=z^{0}$. Таким образом, лемма 4.2 доказана.

Tеорема 4.3. Eсли $f \in M^{0}(X)$, то множсество $\operatorname{Per}(f)$ замкнуто.

ДоказАтельство. Предположим противное. Тогда существует точка $z^{0} \in$ $\overline{\operatorname{Per}(f)} \backslash \operatorname{Per}(f)$. Обозначим $\left\{z_{j}\right\}_{j} \geqslant 1$ последовательность периодических точек, сходящуюся к $z^{0}$. Так как ord $z^{0}$ конечен, то найдется подпоследовательность 
$\left\{z_{j_{i}}\right\}_{i \geqslant 1} \subset\left\{z_{j}\right\}_{j \geqslant 1}$, все точки которой принадлежат одной компоненте $X_{k_{1}}\left(z^{0}\right)$ точки $z^{0}$. В силу леммы 4.2 найдется последовательность $\left\{x_{i}\right\}_{i \geqslant 1} \subset \operatorname{Per}(f) \cap$ $X_{k_{1}}\left(z^{0}\right)$ такая, что $\lim _{i \rightarrow \infty} x_{i}=z^{0}$ и $x_{i} \in \gamma\left(z^{0}, z_{1}\right), i \geqslant 1$. Из теоремы 4.1 следует существование окрестности $U\left(z^{0}\right)$ точки $z^{0}$, для которой $U\left(z^{0}\right) \cap\left\{f^{i}\left(z^{0}\right)\right\}_{i \geqslant 1}=\varnothing$. Тогда найдется натуральное $i_{0} \geqslant 1$ такое, что при любом $i \geqslant i_{0} x_{i} \in U\left(z^{0}\right)$. Так как $X$ - локально связный континуум и $\lim _{i \rightarrow \infty} x_{i}=z^{0}$, то укажем $i_{1} \geqslant i_{0}$ такое, что при любом $i \geqslant i_{1}$ в случае ord $x_{i} \geqslant 3$ для каждой компоненты $X_{s}\left(x_{i}\right)$ точки $x_{i}$, для которой справедливо $X_{s}\left(x_{i}\right) \cap \gamma\left(z^{0}, z_{1}\right)=\varnothing$, вьполнено включение $X_{s}\left(x_{i}\right) \subset U\left(z^{0}\right)$. Возьмем произвольно и зафиксируем $i>i_{1}$. Обозначим через $m_{i}$ период точки $x_{i}$, через $X_{s_{1}}\left(x_{i}\right)\left(X_{s_{2}}\left(x_{i}\right)\right)$ - компоненту точки $x_{i}$, содержашую $z^{0}\left(z_{1}\right)$. Рассмотрим $f^{m_{i}}\left(\gamma\left(z^{0}, x_{i}\right)\right)$. Тогда в силу выбора окрестности $U\left(z^{0}\right)$ и номера $i>i_{1}$ реализуется один из следующих двух случаев: либо $f^{m_{i}}\left(z^{0}\right) \in X_{s_{1}}\left(x_{i}\right)$, либо $f^{m_{i}}\left(z^{0}\right) \in X_{s_{2}}\left(x_{i}\right)$. Пусть выполнено первое условие. Так как $f^{m_{i}}\left(z^{0}\right) \notin U\left(z^{0}\right)$, то $\gamma\left(z^{0}, x_{i}\right) \subset f^{m_{i}}\left(\gamma\left(z^{0}, x_{i}\right)\right)$. Отсюда следует, что для некоторого $j>i x_{j}, f^{m_{i}}\left(x_{j}\right) \in \gamma\left(f^{m_{i}}\left(z^{0}\right), x_{i}\right)$. Последнее противоречит лемме 2.13. Если имеет место второй случай, то

$$
f^{m}\left(\gamma\left(z^{0}, x_{i}\right)\right) \cap f^{m}\left(\gamma\left(x_{i}, z_{1}\right)\right) \neq\left\{x_{i}\right\},
$$

где $m$ - наименьшее общее кратное периодов $m_{1}$ и $m_{i}$ точек $z_{1}$ и $z_{i}$ соответственно. Полученное противоречие с леммой 2.10 исключает и второй случай. Таким образом, с деланное предположение неверно, и $\operatorname{Per}(f)$ замкнуто. Теорема 4.3 доказана.

СлеДСТвиЕ 4.4. Пусть $f \in M^{0}(X)$. Тогда $\operatorname{Per}(f)=C(f)$.

Таким образом, утверждение (B1) теоремы В доказано.

2. Перейдем к доказательству утверждений (B2) и (В3) теоремы В. Будем предполагать, что $\operatorname{Per}(f) \neq X$ (если $\operatorname{Per}(f)=X$, то утверждения (В2) и (В3) справедливы). Обозначим через $V$ произвольную компоненту открытого множества $X \backslash \operatorname{Per}(f)$.

Лемма 4.5. Пусть $f \in M^{0}(X)$, тогда существует дуга $\gamma(x, y) \subset \bar{V}$ такая, что $x \in \operatorname{Per}(f)$ и $f^{m}(\gamma(x, y)) \subset \gamma(x, y)$ при некотором натуральном $m$, кратном периоду $m(x)$ точки $x$.

ДокАЗАтЕЛЬство. Заметим, что $\operatorname{card}(\bar{V} \cap \operatorname{Per}(f))=1$ или 2.

1. Пусть $\operatorname{card}(\bar{V} \cap \operatorname{Per}(f))=2$, а точки $x, x_{1} \in \bar{V} \cap \operatorname{Per}(f)$ имеют периоды $m(x)$ и $m\left(x_{1}\right)$ соответственно. Если $m$ - наименшшее общее кратное периодов $m(x)$ и $m\left(x_{1}\right)$, то в силу леммы $2.9 f^{m}\left(\gamma\left(x, x_{1}\right)\right)=\gamma\left(x, x_{1}\right)$. Пусть для определенности $x$ - притягивающая точка сужения отображения $f^{m}$ на $\gamma\left(x, x_{1}\right)$. Тогда для любой точки $y \in \gamma\left(x, x_{1}\right) \backslash\left\{x_{1}\right\} f^{m}(\gamma(x, y))$ - собственное подмножество дуги $\gamma(x, y)$.

2. Пусть $\operatorname{card}(\bar{V} \cap \operatorname{Per}(f))=1$, a $x \in \bar{V} \cap \operatorname{Per}(f), m_{1} \geqslant 1$-период $x$. Утверждение леммы справедливо, если существует точка $z \in V$ такая, что при некотором $n_{0} \geqslant 1$ $f^{n_{0}}(z)=x$. Поэтому рассмотрим случай, когда для любой точки $z \in V$ и любого натурального $n \geqslant 1 f^{n}(z) \neq x$. Покажем, что $x-\omega$-предельная точка $\omega(z, g)$ траектории $z$ относительно $g=f^{m_{1}}$. Предположим противное. Тогда найдется точка $\bar{z} \in V$ такая, что $x \notin \omega(\bar{z}, g)$. В силу теоремы Дж. Биркгофа (см. [10]) и следствия 4.4 существует $y \in \operatorname{Per}(f) \backslash\{x\}$ периода $m_{2} \geqslant 1$ такая, что $y \in \omega(\bar{z}, g)$. Пусть $m$ 
- наименьшее обшее кратное периодов $m_{1}$ и $m_{2}$. Рассмотрим множество $\omega\left(\bar{z}, g^{m}\right)$. Тогда найдется точка $y_{1} \in \operatorname{Orb}(y, g)$ такая, что $y_{1} \in \omega\left(\bar{z}, g^{m}\right)$. Следовательно, существует натуральное $n_{0} \geqslant 1$ такое, что $g^{m n_{0}}(\bar{z})$ принадлежит некоторой окрестности $U\left(y_{1}\right)$ точки $y_{1}$ такой, что $U(y) \cap\{x\}=\varnothing$. Так как $\bar{V} \cap \operatorname{Per}(f)=\{x\}$, то $y \notin \bar{V}$. Тогда $g^{m n_{0}}(\gamma(x, y)) \cap g^{m n_{0}}(\gamma(x, \bar{z})) \neq\{x\}$, что противоречит лемме 2.10 . Таким образом, для любой точки $z \in V \quad x \in \omega(z, g)$.

Пусть $z$ - любая точка компоненты $V$. Рассмотрим множество $\left\{g^{s}(z)\right\}_{s=0}^{\text {ord } x}$. Так как порядок ord $x$ точки $x$ конечен и $\operatorname{card}\left(\left\{g^{s}(z)\right\}_{s=0}^{\operatorname{ord} x}\right)=\operatorname{ord} x+1$, то существуют $0 \leqslant s_{1}<s_{2} \leqslant$ ord $x$ такие, что $g^{s_{1}}(z), g^{s_{2}}(z)$ принадлежат одной компоненте $X_{k}(x)$ точки $x$ при некотором $1 \leqslant k \leqslant$ ord $x$. Покажем, что $g^{s_{2}-s_{1}}(V) \subset V$. Утверждение справедливо, если $X_{k}(x)=V$. Пусть $X_{k}(x) \neq V$. Тогда $\overline{X_{k}(x)} \cap \bar{V}=\{x\}$. В силу монотонности $g$ полный прообраз $g^{-s_{1}}\left(X_{k}(x)\right)$ компоненты $X_{k}(x)$ принадлежит некоторой компоненте точки $x$. Так как $g^{-s_{1}}\left(X_{k}(x)\right) \cap V \neq \varnothing$, то отсюда следует, что $g^{-s_{1}}\left(X_{k}(x)\right) \subset V$. Следовательно, $g^{s_{2}-s_{1}}(z) \in V$. Поскольку для любой точки $z \in V$ справедливо $g^{s}(z) \neq x, s \geqslant 1$, то из последнего включения следует, что $g^{s_{2}-s_{1}}(V) \subset V$. Обозначим $\tau=g^{s_{2}-s_{1}}$. Пусть $z \in V$ - конщевая точка дендрита $X$. Тогда для $\tau(\gamma(x, z))$ возможны следующие случаи:

1) $\tau(z) \in \gamma(x, z) \backslash\{z\}$;

2) существует точка $u \in R(X) \cap \gamma(x, z) \backslash\{x, z\}$ такая, что $\gamma(x, z) \cup \gamma(u, \tau(z))$ гомеоморфно 3-оду.

В первом случае $\tau(\gamma(x, z))$ - собственное подмножество $\gamma(x, z)$. Пусть имеет место второй случай. Покажем, что $\tau(u) \in \gamma(x, u) \backslash\{u\}$. Действительно, в противном случае так как $\tau(u) \in \gamma(x, \tau(z))$, то $\gamma(x, u) \subset \tau(\gamma(x, u))$. Отсюда следует, что для любого $j \geqslant 1 \gamma(x, u) \subset \tau^{j}(\gamma(x, u))=\gamma\left(x, \tau^{j}(u)\right)$. Последнее означает, что $x \notin \omega(z, \tau)$. Полученное противоречие с доказанным выше показывает, что $\tau(\gamma(x, u)) \subset \gamma(x, u) \backslash\{u\}$. Лемма 4.5 доказана.

СлЕДСТВИЕ 4.6. Пусть $f \in M^{0}(X), V$ - произвольная компонента множества $X \backslash \operatorname{Per}(f)$. Тогда существует точка $x \in \operatorname{Per}(f)$ такая, что $V \subset W^{s}\left(x, f^{m}\right)$, әде $m$ кратно периоду $m(x)$ точки $x, W^{s}\left(x, f^{m}\right)$ - устойчивое многообразие $x$.

СлЕДСТВИЕ 4.7. Пусть $f \in M^{0}(X)$. Тогда для каждой точки $x \in X$ $\omega(x, f)$ - периодическая орбита.

СлеДСТвиЕ 4.8. Пусть $f \in M^{0}(X)$. Тогда $\operatorname{Per}(f)=\bigcup_{x \in X} \omega(x, f)=\Omega(f)=$ $C(f)$.

3. В этой части работы мы докажем утверждение (B4) теоремы В.

Лемма 4.9. Пусть $f \in M^{0}(X)$. Тогда для произвольной точки $z^{0} \in \operatorname{Per}(f)$ периода $m \geqslant 1$ и любого $\varepsilon>0$ существует $0<\delta=\delta\left(z^{0}, \varepsilon\right)<\varepsilon$ такое, что если $z \in \operatorname{Per}(f)$ u $d\left(z^{0}, z\right)<\delta, \operatorname{mo} d\left(f^{m i}\left(z^{0}\right), f^{m i}(z)\right)<\varepsilon$, где $i=1,2, \ldots ; d-$ метрика в $X$.

ДокАЗАТЕльство. Возьмем произвольно $\varepsilon>0$ и точку $z^{0} \in \operatorname{Per}(f)$. Если $z^{0}$ - изолированная точка $\operatorname{Per}(f)$, то выберем $0<\delta<\varepsilon$ такое, что $U_{\delta}\left(z^{0}\right) \cap \operatorname{Per}(f)=$ $\left\{z^{0}\right\}$.

Рассмотрим случай, когда $z^{0}$ - неизолированная точка компакта $\operatorname{Per}(f)$. Предположим, что множество (наименьших) периодов периодических точек из $U_{\varepsilon}\left(z^{0}\right)$ 
ограничено. Обозначим через $p$ наименњшее общее кратное периодов периодических точек из $U_{\varepsilon}\left(z^{0}\right)$. Для произвольного натурального числа $j$ справедливо следующее представление: $j=p j_{1}+j_{2}$, где $j_{1} \geqslant 0,1 \leqslant j_{2}<p$. Так как $f$ непрерывно, то для данного $\varepsilon>0$ сушествует $0<\delta<\varepsilon$ такое, что $f^{m j}\left(U_{\delta}\left(z^{0}\right) \cap\right.$ $\operatorname{Per}(f))=f^{m j_{2}}\left(f^{m p j_{1}}\left(U_{\delta}\left(z^{0}\right) \cap \operatorname{Per}(f)\right)\right)=f^{m j_{2}}\left(U_{\delta}\left(z^{0}\right) \cap \operatorname{Per}(f)\right) \subset U_{\varepsilon}\left(z^{0}\right)$. Последнее включение влечет за собой справедливость леммы 4.9 в рассматриваемом случае.

Пусть множество (наименњших) периодов периодических точек из $U_{\varepsilon}\left(z^{0}\right)$ не ограничено. Тогда в силу леммы 4.2 сушествует последовательность $\left\{z_{j}\right\}_{j \geqslant 1} \subset$ $\operatorname{Per}(f) \cap R(X)$, удовлетворяющая следующим условиям:

1) $\lim _{j \rightarrow \infty} z_{j}=z^{0}$

2) $\left\{z_{j}\right\}_{j \geqslant 1} \subset \gamma\left(z^{0}, z_{1}\right)$

3) существуют компоненты $X_{l\left(z_{j}\right)}\left(z_{j}\right), 1 \leqslant l\left(z_{j}\right) \leqslant \operatorname{ord} z_{j}$, такие, что

$X_{l\left(z_{j}\right)}\left(z_{j}\right) \cap\left\{z_{k}\right\}_{k \geqslant 1}=\varnothing$ и $\bigcup_{j=1}^{\infty} \bigcup_{l\left(z_{j}\right)=1}^{\text {ord } z_{j}-2} X_{l\left(z_{j}\right)}\left(z_{j}\right)$ содержит периодические точки, множество (наименьших) периодов которых не ограничено.

Так как множество $\operatorname{Per}(f)$ замкнуто, то из условия 2) следует, что множество периодов периодических точек $z_{j}, j \geqslant 1$, ограничено. Тогда для данного $\varepsilon>0$ сушествует $0<\delta^{*}<\varepsilon$ такое, что $f^{m i}\left(U_{\delta^{*}}\left(z^{0}\right) \cap\left\{z_{j}\right\}_{j \geqslant 1}\right) \subset U_{\varepsilon}\left(z^{0}\right)$, где $i=1,2, \ldots$. Так как $X-$ локально связный континуум и $X_{l\left(z_{j}\right)}\left(z_{j}\right) \cap\left\{z_{k}\right\}_{k \geqslant 1}=\varnothing$, то $\lim _{j \rightarrow \infty} \operatorname{diam} X_{l_{i}}\left(z_{j}\right)=0$, здесь $\operatorname{diam}(\cdot)$ - диаметр множества $(\cdot)$. Следовательно, сушествует натуральное число $j_{0} \geqslant 1$ такое, что для любого $j>j_{0}$ $f^{m i}\left(X_{l\left(z_{j}\right)}\left(z_{j}\right) \cap \operatorname{Per}(f)\right) \subset U_{\varepsilon}\left(z^{0}\right), i=0,1, \ldots$ Поэтому выберем $0<\delta \leqslant \delta^{*}$ так, чтобы $U_{\delta}\left(z^{0}\right) \cap\left\{z_{j}\right\}_{j=1}^{j_{0}}=\varnothing$. Тогда $f^{m i}\left(U_{\delta}\left(z^{0}\right) \cap \operatorname{Per}(f)\right) \subset U_{\varepsilon}\left(z^{0}\right), i=0,1, \ldots$ Лемма 4.9 доказана.

СлеДСТВИЕ 4.10. Пусть $f \in M^{0}(X)$. Тогда для любой точки $z^{0} \in \operatorname{Per}(f) u$ для любого $\varepsilon>0$ существует $0<\delta=\delta\left(z^{0}, \varepsilon\right)<\varepsilon$ такое, что если $z \in \operatorname{Per}(f)$ $u d\left(z^{0}, z\right)<\delta, \operatorname{mo} d\left(f^{i}\left(z^{0}\right), f^{i}(z)\right)<\varepsilon, i=1,2, \ldots$.

Лемма 4.11. Пусть $f \in M^{0}(X)$. Тогда для любого $\varepsilon>0$ существует $\delta=\delta(\varepsilon)$, удовлетворяющее следующему условию: если $z_{1}, z_{2}$ - произвольнье периодические точки такие, что $d\left(z_{1}, z_{2}\right)<\delta, \operatorname{mo~} d\left(f^{i}\left(z_{1}\right) f^{i}\left(z_{2}\right)\right)<\varepsilon$ для любого $i \geqslant 1$.

ДокАЗАтЕЛьство. Пусть $\varepsilon-$ произвольное положительное число. В силу следствия 4.10 для произвольной точки $z^{0} \in \operatorname{Per}(f)$ и данного $\varepsilon / 2$ существует $0<\delta_{z^{0}}=\delta\left(z^{0}, \varepsilon / 2\right)<\varepsilon / 2$ такое, что если $z \in \operatorname{Per}(f)$ и $d\left(z^{0}, z\right)<\delta_{z^{0}}$, то $d\left(f^{i}\left(z^{0}\right), f^{i}(z)\right)<\varepsilon / 2$. Пусть $\left\{U_{\delta_{z_{0}} / 2}\left(z^{0}\right)\right\}_{z^{0} \in \operatorname{Per}(f)}$ - открытое покрытие множества $\operatorname{Per}(f)$. Так как $\operatorname{Per}(f)$ - компакт, то выберем его конечное подпокрытие $\left\{U_{\delta_{j} / 2}\left(z^{j}\right)\right\}_{j=1}^{n}$. Обозначим $\delta=\min _{1 \leqslant j \leqslant n}\left(\delta_{j} / 2\right)$. Возьмем любые точки $z_{1}, z_{2} \in \operatorname{Per}(f)$ такие, что $d\left(z_{1}, z_{2}\right)<\delta, z_{1} \neq z_{2}$. Тогда сушествует $U_{\delta_{j_{0}} / 2}\left(z^{j_{0}}\right)$ $\left(1 \leqslant j_{0} \leqslant n\right)$ такое, что $z_{1} \in U_{\delta_{0} / 2}\left(z^{j_{0}}\right)$. Поэтому $d\left(z^{j_{0}}, z_{2}\right) \leqslant d\left(z^{j_{0}}, z_{1}\right)+$ $d\left(z_{1}, z_{2}\right)<\delta_{j_{0}} / 2+\delta \leqslant \delta_{j_{0}} / 2+\delta_{j_{0}} / 2=\delta_{j_{0}}$. Следовательно, $d\left(f^{i}\left(z_{1}\right), f^{i}\left(z_{2}\right)\right) \leqslant$ $d\left(f^{i}\left(z_{1}\right), f^{i}\left(z^{j_{0}}\right)\right)+d\left(f^{i}\left(z^{j_{0}}\right), f^{i}\left(z_{2}\right)\right)<\varepsilon / 2+\varepsilon / 2=\varepsilon$. Лемма 4.11 доказана.

Пусть $d_{i}(x, y)=\max _{1 \leqslant j \leqslant i} d\left(f^{j}(x), f^{j}(y)\right)$ - метрика в компакте $\operatorname{Per}(f), i \geqslant 0$. 
Обозначим через $r_{i}(f, \varepsilon), i \geqslant 1$, минимальное число элементов $\varepsilon$-сети множест-

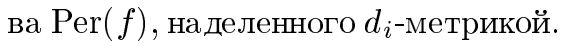

Возьмем произвольно $\varepsilon>0$. В силу леммы 4.11 существует $\delta=\delta(\varepsilon)>0$ такое, что следуюшие условия эквивалентны: 1) конечное множество $Y$ является $\varepsilon$-сетью

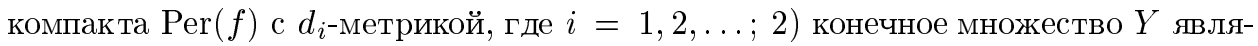
ется $\delta$-сетью множества $\operatorname{Per}(f)$ с $d_{0}$-метрикой. Поэтому справедливо следуюшее утверждение.

Лемма 4.12. Пусть $f \in M^{0}(X)$ u $\varepsilon>0$. Тогда существует $\delta=\delta(\varepsilon)>0$ такое, что $r_{0}(f, \delta)=r_{i}(f, \varepsilon)$ для любого $i=1,2, \ldots$.

Из леммы 4.12 следует, что $h(f)=\lim _{\varepsilon \rightarrow 0} \varlimsup_{\lim _{i \rightarrow+\infty}} \frac{1}{i} \ln r_{i}(f, \varepsilon)=0$. Таким образом, утверждение (B4) теоремы В доказано. Теорема В доказана полностью.

Завершая эту часть работы, покажем, что для любого неограниченного подмножества $M=\left\{m_{1}<\cdots<m_{i}<\ldots\right\}_{i \geqslant 1}$ множества натуральных чисел существуют дендрит $X$ со счетным множеством точек ветвления и непрерывное отображение $f: X \rightarrow X$ такое, что $h(f)=0, T(f)=M$.

ПримеР 4.13. Пусть $O x_{1} x_{2} x_{3}$ - декартова система координат в пространстве $\mathbb{R}^{3}$. Возьмем произвольно последовательность натуральных чисел $3 \leqslant n_{0} \leqslant$ $n_{1}<\cdots<n_{j}<\cdots$. Обозначим через $X_{0}^{\left(n_{j}\right)} n_{j}$-од в плоскости $x_{3}=0$, где $j \geqslant 0$. Положим $X^{\left(n_{0}\right)}=X_{0}^{\left(n_{0}\right)} ; X^{\left(n_{j}\right)}=\left\{\left(x_{1} / j, x_{2} / j, 1 / j\right):\left(x_{1}, x_{2}, 0\right) \in X_{0}^{\left(n_{j}\right)}\right\}$, если $j \geqslant 1$. Определим дендрит $X=X^{(0)} \cup \bigcup_{j=0}^{+\infty} X^{\left(n_{j}\right)}$, где $X^{(0)}=\left\{\left(0,0, x_{3}\right): 0 \leqslant\right.$ $\left.x_{3} \leqslant 1\right\}$.

Положим

$$
n_{j}= \begin{cases}3, & \text { если } j=0, m_{1}=1 \text { или } 2 ; \\ m_{1}, & \text { если } j=0, m_{1} \geqslant 3 ; \\ m_{j+1}, & \text { если } j \geqslant 1 .\end{cases}
$$

Определим отображение $f \in M^{0}(X)$ следующим образом:

1) $\left.f\right|_{X^{(0)}}=\left.\mathrm{id}\right|_{X^{(0)}}$

2) если $j=0, m_{1}=1$ или 2 , то $\left.f\right|_{X_{1}}=\left.\mathrm{id}\right|_{X_{1}},\left.f^{2}\right|_{X_{2} \cup X_{3}}=\left.\mathrm{id}\right|_{X_{2} \cup X_{3}}$, $f\left(X_{2}\right)=X_{3}, f\left(X_{3}\right)=X_{2}$, где $X_{j}$-ветвь триода $X^{\left(n_{0}\right)}$, угловая координата произвольной точки которой равна $(2 \pi / 3)(j-1), j=1,2,3$.

3) если $j=0, m_{1} \geqslant 3$, то $\left.f\right|_{X^{\left(n_{0}\right)}}-$ поворот на угол $2 \pi / n_{0}$;

4) если $j \geqslant 1$, то $\left.f\right|_{X^{\left(n_{j}\right)}}-$ поворот на угол $2 \pi / n_{j}$ в плоскости $x_{3}=1 / j$.

Тогда $T(f)=\{1, M\}$. Из утверждения (B4) теоремы В следует, что $h(f)=0$. В частности, можно положить $M=\{1, m j\}_{j \geqslant 1}$ или $M=\left\{p^{j}\right\}_{j \geqslant 0}$, где $m$ - произвольное натуральное, а $p \geqslant 2$ - произвольное простое число. Если $\rho(M)$ - верхняя плотность множества $M$, т.е. $\rho(M)=\lim _{n \rightarrow \infty} \sup \frac{1}{n} \operatorname{Card}\{k \in M: k \leqslant n\}$, то для множества $M=\{1, m j\}_{j \geqslant 1}$ справедливо $\rho(M)>0$. Таким образом, критерий равенства 0 (положительности) топологической энтропии непрерывного отображения конечного дерева в себя [11] (соответственно [12], [13]) не допускает обобщения на случай непрерывных отображений дендритов со счетным множеством точек ветвления. 


\section{§5. Доказательство теоремы C}

Определим конструктивно дендрит $X \notin D$ и монотонное отображение на нем, не обладаюшее свойствами (B1)-(В3), указанными в теореме В.

I. Построение дендрита. Пусть $\Delta$ - равносторонний треугольник со стороной 1 , лежаший в первом квадрантеплоскости $O x_{1} x_{2}$, основанием которого служит отрезок $[0,1]$ оси $O x_{1}$. Разобьем две его боковые стороны на три равные части и каждый конец интервала $\left(\frac{1}{3}, \frac{2}{3}\right) \subset[0,1]$ соединим с ближайшей точкой деления. Получим два равносторонних треугольника $\Delta_{0}, \Delta_{1}$, опирающиеся на отрезки $\left[0, \frac{1}{3}\right],\left[\frac{2}{3}, 1\right]$ соответственно и пятиугольник, две смежные стороны которого $\gamma_{0}, \gamma_{1}$ принадлежат боковым сторонам исходного треугольника, левой и правой соответственно. Удалив внутренность пятиугольника и интервал $\left(\frac{1}{3}, \frac{2}{3}\right)$, получим замкнутое множество $\Delta^{(1)} \subset \Delta$, состоящее из $\gamma_{0}, \gamma_{1}$ и $\Delta_{0}, \Delta_{1}$. С каждым из треугольников $\Delta_{0}, \Delta_{1}$ повторим то же построение. Получим замкнутое множество $\Delta^{(2)} \subset \Delta^{(1)}$, состоящее из дуг $\gamma_{0}, \gamma_{1}, \gamma_{00}, \gamma_{01}, \gamma_{10}, \gamma_{11}$ и треугольников $\Delta_{00}, \Delta_{01}, \Delta_{10}, \Delta_{11}$. Продолжая построение, для любого $n \geqslant 2$ получим замкнутое множество $\Delta^{(n)} \subset \Delta^{(n-1)}$, которое является объединением $2^{n}$ треугольников $\Delta_{i_{1} \ldots i_{n}}$ (каждый из индексов $i_{1}, \ldots, i_{n}$ принимает значение 0 и 1$)$, дуг множества $\Delta^{(n-1)}: \gamma_{i_{1} \ldots i_{k}}, 1 \leqslant k \leqslant n-1$, и дуг $\gamma_{i_{1} \ldots i_{n}}\left(i_{1}, \ldots, i_{n}\right.$ принимают значения 0 и 1$)$. Таким образом, построена последовательность континуумов $\Delta \supset \Delta^{(1)} \supset \Delta^{(2)} \supset \cdots \supset \Delta^{(n)} \supset \cdots$. Тогда множество $X=\bigcap_{n \geqslant 1} \Delta^{(n)}$ есть континуум $[7 ; \S 47$, п. 2]. Легко проверить, что $X$ - локально связный континуум, не содержащий путей, гомеоморфных окружности. Следовательно, $X$ - дендрит, множество концевых точек $E(X)$ которого есть канторов дисконтинуум, принадлежащий отрезку [0, 1$]$.

II. Построение отображения. Обозначим через $X_{n}=\bigcup_{i_{1}, \ldots, i_{n} \in\{0,1\}} \gamma_{i_{1} \ldots i_{n}}$, $n \geqslant 1$, через $o$ - вершину треугольника $\Delta$, не принадлежашую основанию $[0,1]$. Определим отображение $f \in M^{0}(X)$ следующим образом.

1. $\left.f\right|_{X_{1}}=\tau$, где $\tau$ - “связка поворотов" такая, что $\tau(o)=o$ и для любого $x \in X_{1} \backslash\{o\} d(o, x)=d(o, \tau(x))$, причем $\tau(x) \neq x$.

2. При любом $n \geqslant 2$ положим

$$
X_{n}^{(0)}=\bigcup_{i_{2} \ldots i_{n} \in\{0,1\}} \gamma_{0 i_{2} \ldots i_{n}}, \quad X_{n}^{(1)}=\bigcup_{i_{2} \ldots i_{n} \in\{0,1\}} \gamma_{1 i_{2} \ldots i_{n}}
$$

Определим $f$ на $X_{n}$ таким образом, чтобы $\left.f^{2}\right|_{X_{n}^{\left(i_{1}\right)}}\left(i_{1}=0\right.$ или 1$)$ с точностью до масштабного преобразования с коэффициентом $\frac{1}{3}$ совпадало с $\left.f\right|_{X_{n-1}}$. Тогда $T\left(\left.f\right|_{X_{n}}\right)=\left\{2^{n-1}, 2^{n}\right\}, n \geqslant 1$.

3. Для любой точки $y \in E(X)$ положим

$$
f(y)=\lim _{n \rightarrow \infty} f\left(x_{n}\right),
$$

где $\left\{x_{n}\right\}_{n \geqslant 1}$ - произвольная последовательность, сходящаяся к $y$.

Из определения $f$ следует, что последовательность $\left\{f\left(x_{n}\right)\right\}_{n \geqslant 1}$ фундаментальна. Следовательно, предел (1) существует. Покажем его независимость от выбора последовательности $\left\{x_{n}\right\}_{n \geqslant 1}$. Пусть $\left\{x_{n}\right\}_{n \geqslant 1},\left\{\bar{x}_{m}\right\}_{m \geqslant 1}-$ различные последовательности точек, сходящиеся к $y$. Тогда для любого $\varepsilon>0$ сушествует 
$n_{0} \geqslant 1$ такое, что при любых $n, m \geqslant n_{0} d\left(x_{n}, \bar{x}_{m}\right)<\varepsilon$. Из определения $f$ на $\bigcup_{n \geqslant 1} X_{n}$ следует, что $d\left(f\left(x_{n}\right), f\left(\bar{x}_{m}\right)\right)=d\left(x_{n}, \bar{x}_{m}\right)<\varepsilon$. Таким образом, $\lim _{n \rightarrow \infty} f\left(x_{n}\right)=\lim _{n \rightarrow \infty} f\left(\bar{x}_{m}\right)$.

Покажем, что

$$
f(E(X))=E(X)
$$

Пусть $y \in E(X)$, а $\left\{x_{n}\right\}_{n \geqslant 1}-$ произвольная последовательность точек, сходящаяся к $y$. Для каждого $x_{n}, n \geqslant 1$, существует множество $X_{k_{n}}$ такое, что $x_{n} \in$ $X_{k_{n}}$. Из задания $f$ на $X_{k_{n}}$ следует, что $f^{-1}\left(x_{n}\right)$ - одноточечное множество и $f^{-1}\left(x_{n}\right), f\left(x_{n}\right) \in X_{k_{n}}$. Из условия: $\lim _{n \rightarrow \infty} \operatorname{diam} X_{k_{n}}=0$ вытекает справедливость равенства (2).

Заметим, что $f: X \rightarrow X$ - гомеоморфизм. Действительно, по определению $\left.f\right|_{\bigcup_{n \geqslant 1} X_{n}}$ взаимно однозначное и взаимно непрерывное отображение. Используя равенства (1) и (2), получаем отсюда, что $\left.f\right|_{E(X)}$ взаимно однозначно и взаимно непрерывно. Таким образом, $f \in M^{0}(X)$.

Покажем, что для любой точки $y \in E(X) y=\lim _{n \rightarrow \infty} f^{2^{n}}(y)$. Рассмотрим произвольную $\varepsilon$-окрестность $U_{\varepsilon}(y)$ точки $y$. Тогда найдется натуральное $n_{0} \geqslant 1$ такое, что при любом $n \geqslant n_{0} U_{\varepsilon}(y) \cap X_{n} \neq \varnothing$. Возьмем произвольно и зафиксируем $n>n_{0}$. Пусть $\left\{x_{k}\right\}_{k \geqslant 1} \subset U_{\varepsilon}(y)$ - произвольная последовательность точек, сходящаяся к $y$. Сушествует натуральное число $k_{1} \geqslant 1$ такое, что при любом $k \geqslant k_{1} d\left(y, x_{k}\right)<\varepsilon / 3$. В силу определения $f$ получаем отсюда, что $d\left(f^{2^{n}}\left(x_{k}\right), f^{2^{n}}(y)\right)<\varepsilon / 3$. Так как $\left.f^{2^{n}}\right|_{\bigcup_{i=1}^{n} X_{i}}=\left.\mathrm{id}\right|_{\bigcup_{i=1}^{n} X_{i}}$ (где $\mathrm{id}(\cdot)$ - тождественное отображение множества $(\cdot))$, то существует натуральное число $k_{0} \geqslant k_{1}$ такое, что при любом $k \geqslant k_{0} d\left(x_{k}, f^{2^{n}}\left(x_{k}\right)\right)<\varepsilon / 3$. Тогда $d\left(y, f^{2^{n}}(y)\right)<d\left(y, x_{k}\right)+d\left(x_{k}, f^{2^{n}}\left(x_{k}\right)\right)+d\left(f^{2^{n}}\left(x_{k}\right), f^{2^{n}}(y)\right)<\varepsilon$. Отсюда следует, что $y \in \operatorname{Rec}(f) \backslash \operatorname{Per}(f)$, где $\operatorname{Rec}(f)$ - множество рекуррентных точек $f$, и множество периодических точек $\operatorname{Per}(f)=\bigcup_{n \geqslant 1} X_{n}$ незамкнуто. Теорема С доказана.

ЗАмЕчАниЕ 5.1. Незначительно модифицируя построенное отображение, можно указать монотонное отображение, имеющее единственную отталкивающую неподвижную точку о и глобальный аттрактор $E(X)$.

Основные результаты работы анонсированы в [14], [15].

\section{Список литературы}

1. Ayres $W$. Some generalizations of the Scherrer fixed point theorem // Fund. Math. 1930. V. 16. P. 332-336.

2. Li Sh., Ye X. Topological entropy for finite invariant subsets of $Y / /$ Trans. Amer. Math. Soc. 1995. V. 347. № 12. P. 4651-4661.

3. Kato $H$. A note on periodic points and reccurent points of maps of dendrites // Bull. Austral. Math. Soc. 1995. V. 51. № 3. P. 459-461.

4. Kato H. The depth of centres of maps on dendrites // J. Austral. Math. Soc. Ser. A. 1998. V. 64. P. 44-53.

5. Войнова М.И., Ефремова Л. С. О динамике простейших отображений дендритов // Матем. заметки. 1998. Т. 63. №2. С. 183-195.

6. Ефремова Л. С., Махрова E.Н. О динамике монотонных отображений $n$-ода // Изв. вузов. Сер. матем. 1997. № 10. С. 31-36.

7. Куратовский К. Топология. Т. 2. М.: Мир, 1969.

8. Levin $M$. Hyperspaces and open monotone maps of hereditarily indecomposable continua // Proc. Amer. Math. Soc. 1997. V. 125. № 2. P. 603-609. 
9. Smith Hal L. Monotone dynamical systems: an introduction to the theory of competitive and cooperative systems. Providence, RI: Amer. Math. Soc., 1995.

10. Биркгоф Дж. Д. Динамические системы. Ижевск: Изд. дом "Удмуртский университет", 1999.

11. Alseda L., Ye X. No division and the set of periods for tree maps // Ergodic Theory Dynam. Systems. 1995. V. 15. №2. P. 221-237.

12. Llibre J., Misiurewicz M. Horseshoes, entropy and periods for graph maps // Topology. 1993. V. 32. P. 649-664.

13. Blokh A. M. Periods implying almost all periods, trees with snowflakes and zero entropy maps // Nonlinearity. 1992. V. 5. P. 1375-1382.

14. Efremova L. C., Makhrova E. N. On the structure and dynamics of the finite trees monotonic mappings // Proc. 3th Internat. Conf. "Differential Equations and Applications". Saransk, 1998. P. 134-135.

15. Efremova L.S., Makhrova E.N. On dynamics of monotone mappings of dendrites // Proc. Internat Conf. dedicated to the 90th anniversary of L. S. Pontryagin. Moscow, 1998. P. 31-32.

Нижегородский государственный университет им. Н.И. Лобачевского

E-mail: efr@mm.unn.ac.ru, mak@mm.unn.ac.ru
Поступила в редакцию 21.10 .1999 и 25.12 .2000 